\title{
Introducing Service Differentiation in a Bluetooth Piconet
}

\author{
Antoine Mercier ${ }^{1}$ and Pascale Minet ${ }^{2}$ \\ 1 Ecole Centrale d'Electronique, LACCSC, 53 rue de Grenelle, 75007 Paris, France \\ mercier@ece.fr \\ 2 INRIA, Rocquencourt, 78153 Le Chesnay, France \\ pascale.minet@inria.fr
}

\begin{abstract}
In this paper, we focus on the Bluetooth wireless network, analyzing its ability to support Quality of Service (QoS) requirements defined by the application. We focus on two QoS parameters : (i) an application constraint denoting the importance degree of a message, and (ii) an end-to-end delivery deadline. In a first step, we introduce a local scheduling accounting for the two QoS parameters in One-Round Robin (1-RR). We evaluate the performances of this enhanced version. In a second step, we define a new Bluetooth global scheduling, called ClassBased Earliest Deadline First (CB-EDF). It takes into account the two QoS parameters at the global level. Simulation results show that CBEDF achieves a better service differentiation than enhanced 1-RR and allows the coexistence of messages with different application constraints.
\end{abstract}

\section{Introduction}

Bluetooth [1] is defined as a solution for ad-hoc networking using polling scheme as medium access control. Bluetooth devices are organized into piconets where a central device acts as the master, which solicits packets transmission from up to seven active slaves. In this paper, we study how Bluetooth devices account for Quality of Service (QoS) requirements, expressed by QoS sensitive applications. More precisely, we focus on the scheduling mechanisms used to support these QoS requirements. We distinguish two message scheduling mechanisms:

- Local scheduling refers to the mechanism selecting one message in the local waiting queues of the Bluetooth device considered.

- Intra-piconet scheduling refers to the polling scheme within a piconet. It is defined in [3] as the set of rules that determines when the piconet master switches from one slave to another. The combination of local and intrapiconet schedulings is referred to as global scheduling.

In order to support QoS applications, real-time constraints must be accounted for by both local and intra piconet schedulings. We report simulations of the native One-Round Robin (1-RR). We focus on the support of two application constraints: (i) the first one denoting the importance degree of a message, and (ii) the second referring to the message delivery deadline. As expected, 1-RR does not achieve service differentiation: 
- High importance messages are transmitted after low importance ones.

- Messages with close deadlines are transmitted after messages with the same importance and far deadlines.

To improve performances of 1-RR, we introduce a local scheduling that takes into account these two constraints by a combination of class based Priority Queuing (PQ) - a class contains all the messages of the same importance degree - and Earliest Deadline First (EDF). PQ is used between classes and EDF is used within a class. This local improvement allows a better service differentiation. However, it does not guarantee that the message belonging to the highest priority class is transmitted first. To achieve that, classes and deadlines have to be accounted for by the intra-piconet scheduling. We then design an optimal solution technically unrealizable, where the master has a complete knowledge of pending messages in the piconet. This solution is uniquely given as a reference for comparative evaluation. We finally define a global scheduling mechanism, called Class-Based Earliest Deadline First (CB-EDF), that supports the two previous application constraints. Performances of CB-EDF are evaluated by simulations.

This paper is organized as follows. In Section 2, we consider the local and intra-piconet problem and present a brief state of the art describing the main polling schemes. In section 3 , we introduce into 1-RR a local scheduling accounting for classes and deadlines. Performances of this enhanced version are compared with those of the native one. In Section 4, we present our solution, CB-EDF and compare its performances with those of the optimal solution.

\section{State of the Art}

In this section, we present a brief state of the art of local and intra-piconet schedulings and give a classification of main intra-piconet schedulings. In this paper, we consider only Asynchronous ConnectionLess links, because they offer better flexibility [1]. We recall that the master maintains at least one queue per slave, denoted $M \rightarrow S$ and each slave maintains at least a slave-to-master queue, denoted $S \rightarrow M$.

\subsection{Local Scheduling}

Bluetooth specifications [1] recommend the use of FIFO for local scheduling. We preconize here the use of classes and deadlines to account for the message importance and deadline. The local scheduling is then class based. Moreover, we recommend the use of EDF algorithm within each class, as it has been proved optimal for a uniprocessor scheduling in both preemptive and non-preemptive context when the message arrival times are not known a priori ${ }^{1}$.

${ }^{1}$ K. Jeffay, D. F. Stanat, C. U. Martel: On non-preemptive scheduling of periodic and sporadic tasks, IEEE Real-Time Systems Symposium (1991), San-Antonio, Texas. 


\subsection{Intra Piconet Scheduling}

Polling schemes designed or extended for Bluetooth technology can be classified according to three main criteria. The first criterion allows determining which slaves are polled. In full polling mechanism such as 1-RR or ERR [3], all slaves in the piconet, even if they have nothing to transmit, are polled. In activity based polling (e.g. FEP in [4]), the polling cycle can be adjusted according to the activity of each slave. Slaves with empty queues are less frequently polled to prevent the bandwidth wastage. The second criterion corresponds to the slave polling order. This order can be determined to achieve fairness access between slaves or to maximize throughput. Static or dynamic priority can be assigned to each slave to determine the polling order. This priority can be mapped:

- In case of full polling:

- arbitrarily: e.g. arrival order of link requests (e.g. 1-RR and ERR in [3]).

- from slave parameters: e.g. the poll interval negotiated during the link establishment or the $M \rightarrow S$ queue length (e.g. EPM in [3]).

- In case of activity based polling:

- arbitrarily: e.g. FEP in [4].

- from slave parameters: bandwidth requirements (e.g. PFP in [5], FPQ in [6]), delivery deadline (eg. FPQ in [6]), $M \rightarrow S$ or $S \rightarrow M$ queue length (e.g. AFP and SAFP in [7]), size of the Head-Of-Line message (e.g. PP and $\mathrm{KFP}$ in [8]).

The third criterion is related to the use of information about the $S \rightarrow M$ queues. If used, such information is provided by a signalling scheme or a prediction algorithm. With a signalling scheme for $S \rightarrow M$ queue, slaves can communicate their queue status while transmitting packets. Such information can be included into a specific packet field (e.g. [7] uses the flow bit in the header). With a prediction algorithm, the master estimates the message presence in $S \rightarrow M$ queues. This prediction is based on the arrival laws of messages on a slave (e.g. $\mathrm{PFP}$ in [5], FPQ in [6]).

According to this classification, CB-EDF is based on an activity based polling; the priority of a slave is deduced from its message parameters (class and delivery deadline); CB-EDF uses a combination of signalling and prediction schemes.

\section{Service Differentiation with 1-RR}

In this section, we evaluate the performances by simulation of 1-RR with regard to the application constraints. We consider its native version and its version enhanced with local scheduling accounting for message importance and deadline.

\subsection{Local Scheduling Algorithm Principles}

The local scheduling combines class based PQ, a class contains all messages of the same importance degree, and EDF. PQ is used between classes and EDF is 
used within a class. This local scheduling is used by both master and slaves to manage $M \rightarrow S$ or $S \rightarrow M$ queues. Each $S \rightarrow M$ or $M \rightarrow S$ queue consists of $N$ distinct sub-queues; each class $i(i \in[1, N])$ has its own sub-queue. According to PQ principle, messages from class $i+1$ are always transmitted before those from class $i$. In each sub-queue, the transmission order is determined by EDF. This local scheduling will be used with the enhanced version of 1-RR and CB-EDF.

\subsection{Simulation Parameters and Scenario}

Before running the simulations described hereafter, we have validated the simulation tool we have developed, by confrontation with the results already published in [3]. In all scenarios, we consider a piconet made up of 7 slaves. Four classes are managed (class 3 having the highest priority). Each slave generates 2 flows in each of the 4 classes. In the same way, the master has, for each slave, 4 classes with 2 flows per class. Each flow follows a Poisson arrival law. The size of flow messages follows an exponential law of parameter 8, representing the number of slots required for the message transmission. The relative deadlines associated with class 0 and 1 range from 600 to $1000 \mathrm{~ms}$, with class 2 from 400 to $600 \mathrm{~ms}$ and with class 3 from 200 to $400 \mathrm{~ms}$. Each simulation provides the following results:

- For any class, the average response time of any $S \rightarrow M$ flow.

- For any class, the percentage of $S \rightarrow M$ messages missing their deadline.

- The efficiency of the polling scheme. It is computed as the percentage of useful slots over the total slot number.

To perform a comparative performance evaluation, we have considered a scenario representative of Bluetooth communications. The submitted load is equal to $80 \%$. The traffic distribution varies as follows with $n$ ranging from 0 to 10 ; when $n=0$, the distribution is symmetric and uniform :

- $S \rightarrow M$ flows of slaves 1 to 3 are heavily loaded: the submitted load of these flows ranges from $17 \%(n=0)$ to $80 \%(n=10)$.

- The submitted load of $S \rightarrow M$ flows of slaves 4 to 7 and $M \rightarrow S$ flows of slaves 1 to 7 ranges from $63 \%(n=0)$ to $0 \%(n=10)$.

- Class 3 (resp. 2, 1,0) contributes to $10 \%$ of the load (resp. 20\%, 30\%, 40\%).

\subsection{Evaluation of 1-RR}

For 1-RR, the master polls successively each slave for one message. In this section, we evaluate the performances of 1-RR and enhanced 1-RR polling schemes. The average response times of slaves 1 and 4 are illustrated on Fig. 1.

As expected, the initial 1-RR does not provide service differentiation. For a given $n, n \in[0,10]$, each class has the same average response time. For class 3 , the percentage of messages missing their deadline is greater than in other classes; it ranges from $15 \%$ to $100 \%$ when $n$ ranges from 0 to 10 . It can be explained because the highest priority class has smaller deadlines. The efficiency is the same for 1-RR and enhanced 1-RR (eg. $79.6 \%$ for $n=0$ and $47.7 \%$ for $n=10$ ). Enhanced 1-RR only provides an acceptable service differentiation in case of traffic uniformly distributed over the slaves $(n=0)$. 

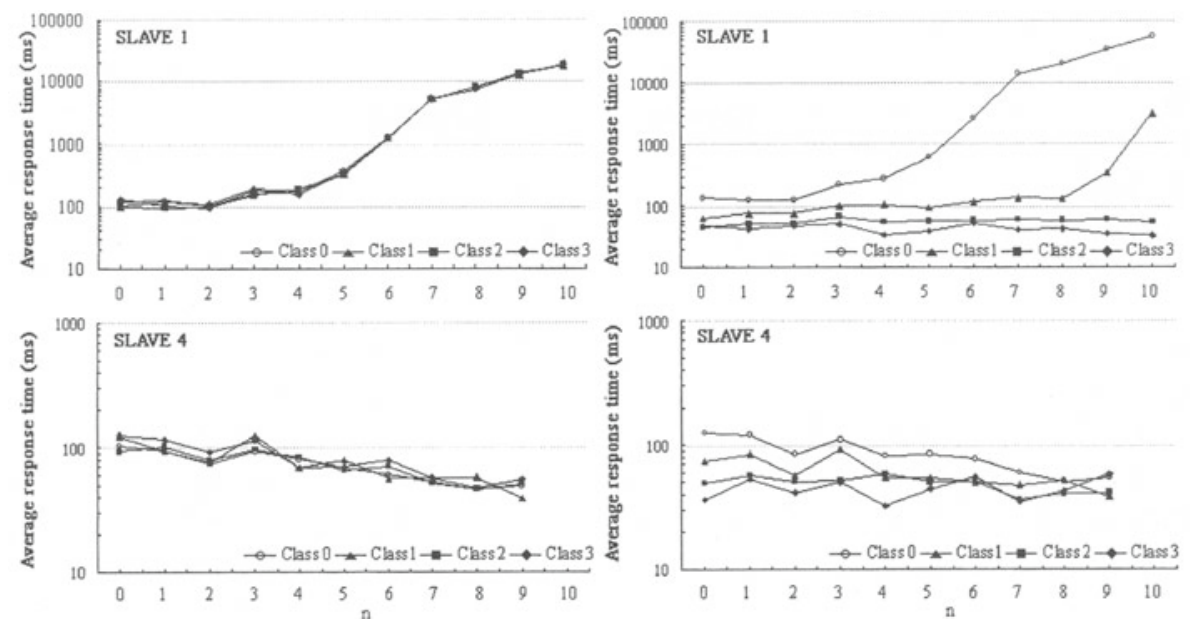

Fig. 1. Simulation for 1-RR and enhanced 1-RR for slaves 1 and 4.

\section{A Class-Based EDF Scheduling}

We present in this section our solution, called CB-EDF, to support QoS in the piconet. We give performance evaluations for the scenario used with 1-RR.

\subsection{Principles}

Our purpose is to define a global scheduling mechanism that supports service differentiation. Our solution accounts for two application constraints:

- the importance degree of a message that is mapped in a class,

- the end-to-end delivery deadline.

These constraints are accounted for and integrated within Bluetooth local and intra-piconet schedulings by a combination of PQ between classes and EDF within a class. We define two versions of this algorithm. The first one corresponds to an optimal solution, where a global and complete knowledge of the messages and their arrival times in each $M \rightarrow S$ and $S \rightarrow M$ queues is assumed. This version has no practical interest, except for comparative evaluation. In considering all $M \rightarrow S$ and $S \rightarrow M$ pending messages, the master always chooses the slave with the highest priority class. In case of equality, the master arbitrates in favor of the slave with the message having the earliest absolute deadline in this class. The second version corresponds to a realistic solution where the knowledge of $S \rightarrow M$ queues is obtained from an original scheme combining signalling and prediction. This version is called CB-EDF. It requires, for any slave, the knowledge of the message arrival laws and deadlines of any class present on this slave. 


\subsection{Message Presence Evaluation}

We now describe the module of CB-EDF in charge of message presence evaluation in $S \rightarrow M$ queues of each active slave on the piconet. The combination of signalling and prediction enables to improve the quality of message presence evaluation. As we assume that messages in a given class can be generated by different application flows, L2CAP [1] mechanism signalling the presence of another segment in queue cannot be used. For that purpose, we propose a slight modification in Bluetooth specifications: we use 2 bits in the header of the Baseband packet to notify the master of pending message at the polled slave [2]. However, this mechanism is unable to signal the presence of messages arrived after the slave poll. That is why, we use a prediction algorithm. This algorithm is based on the knowledge of the message arrival law to estimate the presence of messages arrived in each class of a slave after its last poll.

\subsection{Performance Evaluation of CB-EDF}

In this section, we evaluate by simulation the performance of CB-EDF and compare them with those obtained by the optimal solution.

The results show that CB-EDF provides a good service differentiation in all simulated scenarios, better than enhanced 1-RR. Indeed, average response times for the two highest priority classes are shorter as well as the percentage of messages missing their deadline. The results obtained with CB-EDF are close to those obtained by the optimal solution. The efficiency is the same for CB-EDF and the optimal solution for any $n \in[0,10]$ (eg. $79.8 \%$ for $n=0$ and $76.0 \%$ for $n=10$ ). CB-EDF approximates the global exact knowledge of the optimal solution by combining the signalling scheme with the prediction algorithm.
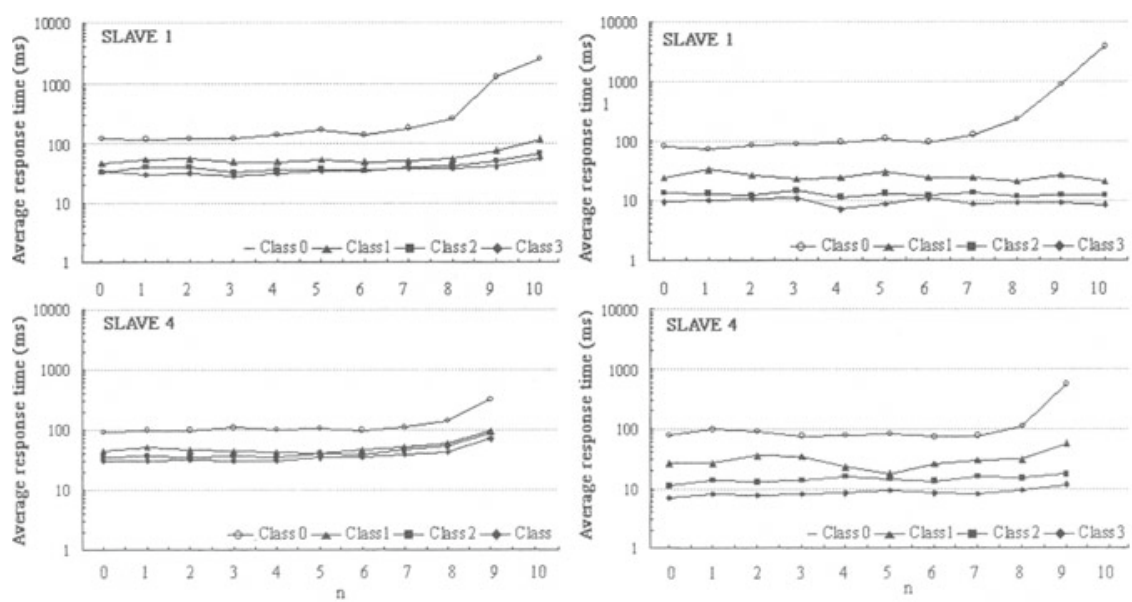

Fig. 2. Simulation for CB-EDF and the optimal solution for slaves 1 and 4 . 


\section{Conclusion}

In this paper, we have shown how to account for QoS in a piconet scheduling. Two QoS parameters, defined by the application, have been considered: the importance of a message and its delivery deadline. The simplest way to introduce service differentiation in a piconet consists in replacing the local scheduling, FIFO, by a scheduling managing classes and deadlines, while keeping the existing intra-piconet scheduling, 1-RR. This solution provides an acceptable service differentiation when the load is uniformly distributed. However, this solution does not account for classes and deadlines in the intra-piconet scheduling. Hence, in a second step, we have proposed CB-EDF that allows the coexistence of messages with different constraints. Simulations results show that in all simulated scenarios, CB-EDF provides service differentiation without efficiency loss and achieves good performances by the combination of signalling and prediction algorithms.

\section{References}

1. Specification of the Bluetooth system, version 1.b, www.bluetooth.com (1999).

2. A. Mercier, P. Minet, L. George: Introducing QoS support in Bluetooth Piconet with a Class-Based EDF Scheduling, INRIA Research report 5054 (2003).

3. A. Capone, M. Gerla, R. Kapoor: Efficient polling schemes for Bluetooth picocells, Proc. of IEEE ICC 2001, Helsinki, Finland.

4. N. J. Johansson, U. Korner, and P. Johansson: Performance evaluation of scheduling algorithms for Bluetooth, in Proc. of IFIP TC6 $5^{\text {th }}$ Int. Conf. on BC'99, Hong-Kong.

5. G. Heijenk, R. A. Yaiz: Predictive Fair Polling, Provisional Application for United States Letters Patent 60/241, 314 (2000).

6. J. B. Lapeyrie, T. Turletti: Adding QoS support for Bluetooth piconet, INRIA Research report 4514 (2002).

7. A. Das, A. Ghose, A. Razdan, H. Saran, R. Shorey: Enhancing performance of asynchronous data traffic over the Bluetooth wireless ad-hoc network, Proc. of IEEE INFOCOM 2001, Alaska, USA.

8. M. Kalia, D. Bansal, R. Shorey: MAC scheduling and SAR policies for Bluetooth a master driven TDD pico-cellular wireless system, in Mobile Multimedia Communications (1999), IEEE Int. Workshop. 